\title{
Non-traditional Approach to the Evaluation of the Freeze-thaw Resistance of Concrete based on the Fracture Tests
}

\author{
Barbara Kucharczyková, ${ }^{1, *}$, Hana Šimonová ${ }^{1}$, Dalibor Kocáb ${ }^{1}$, and Gabriela R. Fernandes ${ }^{2}$ \\ ${ }^{1}$ Brno University of Technology, FCE, Veveří 331/95, Brno, Czech Republic \\ ${ }^{2}$ Federal University of Rio de Janeiro, COPPE/PEC, Rio de Janeiro, Brazil
}

\begin{abstract}
The paper deals with a pilot study focused on the freeze-thaw (F-T) resistance of concrete. A non-traditional approach to the evaluation of the F-T resistance of concrete based on the determination of the fracture parameters is presented in the paper. Three types of specimens were used in the experimental program - prisms with edge and Chevron notch, and cylinders with the Chevron notch. Three sets of specimens of each shape were tested in total. The reference set was stored in the water until the time of testing. Another two sets of specimens were subjected to 50 and 100 freeze-thaw tests, respectively. One F-T cycle consisted of 4 hours freezing in the air at temperature $-18{ }^{\circ} \mathrm{C}$ and 2 hours thawing in the water at temperature $+20^{\circ} \mathrm{C}$. Based on the results, it can be stated that the values of fracture energy are proportional to the area of the ligament. This finding was observed for all tested sets - non-frost and frost attacked. The values of modulus of elasticity and fracture toughness are influenced more by the geometry and also by the changes of the specimens' stiffness due to the exposition to the F-T cycles.
\end{abstract}

\section{Introduction}

The freeze-thaw (F-T) resistance of concrete is one of the crucial parameters related to the durability of concrete structures. The F-T resistance depends on the quality of components and the overall composition of the fresh concrete as well as on the quality of compaction and curing after its placing. In the hardened state, the F-T resistance depends on the quality of the cement matrix, aggregate and interfacial transition zone (ITZ) between the aggregate and cement matrix. The F-T resistance of both components can be verified separately but the mutual interaction and compatibility are crucial for F-T resistance of final concrete.

The F-T resistance depends on the climate conditions which are different over the world. That is why the testing methods intended for the determination of the F-T resistance of concrete are adjusted to the climate conditions of the particular world regions.

*Corresponding author: barbara.kucharczykova@vutbr.cz 
Generally, all of the testing methods are based on the determination of the relative decrease of monitored parameters due to the exposure of the concrete specimens to the F-T cycles. In the case of standardized testing methods, the decrease in tensile and compressive strength or changes, and a decrease in the value of dynamic modulus of elasticity are the most frequently monitored characteristics. Besides, the standards recommend the measurement of the length changes during the F-T test [1,2]. In all cases, the changes in the mass of the test specimens are usually monitored at regular intervals of the F-T tests.

The monitoring of the variations in the fracture characteristics is one of the nonstandardized approaches to the evaluation of the F-T resistance of concrete [3, 4]. The principle of the evaluation consists in the monitoring of the variations in the selected fracture parameters such as fracture toughness, fracture energy, and also variations in the value of modulus of elasticity obtained from the fracture test. The specimens subjected to the fracture tests, performed in a three-point bending test configuration, are usually provided with the central notch which serves as a stress-concentrator positioned in the middle of the specimens' span length. In this way, the position of the potential crack origination is clearly defined which enables the precise measurement of the vertical and horizontal displacement under the crack path. The fracture process of concrete specimens is much more complicated compared to the metals or another homogenous material because of the presence of the aggregate-inclusions and air-pores of different sizes. The presence of nano- and micro-cracks is also very common in concrete specimens [5]. In this context, the shape and depth of the initial notch can influence the fracture behaviour significantly. The central edge or Chevron notch is most frequently employed in the case of brittle and quasibrittle materials testing [6].

In the case of determination of the concrete F-T resistance, prismatic or cylindrical specimens intentionally moulded in the laboratory are usually employed. If the F-T resistance of the existing structure has to be verified, drilled cores can also be used for testing. Nevertheless, this approach is not common for testing the concrete but it is very common for testing the F-T resistance of rock [7].

In this pilot study, two different types of specimens were subjected to the F-T test performed in compliance with the standard ČSN 731322 [8].

\section{Experimental part}

The main aim of performed experiments was to verify the influence of the shape of the test specimens and also the influence of the shape of the initial notch on the resulted fracture characteristics. Both parameters influence the shape and area of the ligament of the test specimens subjected to the fracture test in a three-point bending configuration. Two basic types of specimens were chosen - prisms and cylinders provided with a central edge and Chevron notch.

\subsection{Materials}

Ordinary air-entrained concrete with a maximum aggregate grain size of $16 \mathrm{~mm}$ was used for the manufacturing of the test specimens. CEM I 42.5 R Portland cement (cement plant Mokrá) was used as a binder. The polycarboxylate-based super-plasticizer Sika ViscoCrete (SVC 4035) was used in the amount of $0.26 \%$, air-entraining admixture LPS A 94 in the amount of $0.14 \%$ and workability enhancing admixture Sika Viscoflow $25 \mathrm{CZ}$ in the amount of $0.41 \%$ by cement. The water-cement ratio (w/c) was 0.46 . The concrete was mixed in a concrete plant and transported by the concrete agitator truck to the laboratory. All test specimens including massive blocks were manufactured from the same batch. After compaction, all test specimens were covered with the PE foil to prevent them from 
excessive desiccation. After 48 hours, the test specimens were demoulded and stored in the water bath until the time of the testing.

\subsection{Test specimens}

Two different types of test specimens were manufactured for the experiment - prisms, commonly used for the fracture and F-T tests of concrete, and cylinders, commonly used for the fracture and F-T tests of rocks. Prismatic specimens were moulded into the moulds with the nominal dimensions of $100 \times 100 \times 400 \mathrm{~mm}$. Cylindrical specimens with a diameter of $104 \mathrm{~mm}$ were drilled from the massive concrete blocks with dimensions of 450 $\times 1800 \times 2400 \mathrm{~mm}$ (thickness $\times$ width $\times$ height) and cut into the desired length of $370 \mathrm{~mm}$. For the purpose of the fracture tests, the prismatic specimens were provided with two different shapes of the notch (see Fig. 1 a, b) while keeping the identical area of the ligament. Because the cylindrical specimens are not commonly used for the fracture tests of the concrete, the shape of the notch was designed in compliance with the recommendations given for testing the fracture parameters of the rocks [9].

a)

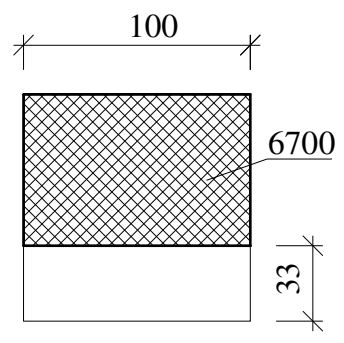

b)

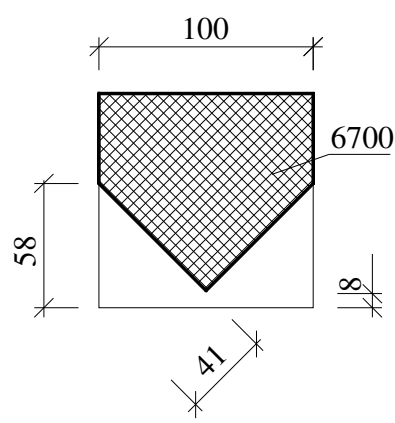

c)

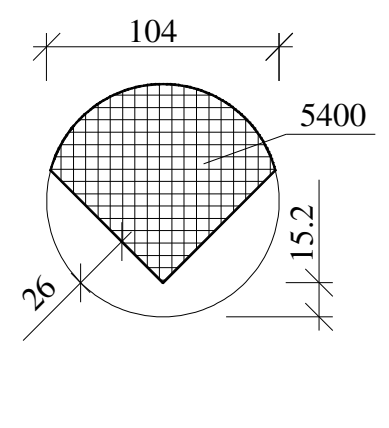

Fig. 1. Shape and area of the ligament of the test specimens: a) Prismatic specimen with edge notch Set_1; b) prismatic specimen with Chevron notch - Set_2, and c) cylindrical specimen with Chevron notch - Set_3.

\subsection{Test methods}

\subsubsection{Freeze-thaw test}

The F-T tests were performed in compliance with the standard ČSN 731322 [8]. One F-T cycle consisted of 4 hours of air-freezing at the temperature of $-18{ }^{\circ} \mathrm{C}$ and 2 hours of thawing in the water at the temperature of $+20{ }^{\circ} \mathrm{C}$. The age of the test specimens at the start of the F-T tests was at least 90 days. All test specimens were fully saturated with water before the start of testing. Each tested set consisted of three groups of test specimens reference (non-frost attacked) specimens, specimens subjected to the $50 \mathrm{~F}-\mathrm{T}$ cycles, and specimens subjected to the $100 \mathrm{~F}-\mathrm{T}$ cycles. Each testing group contained three test specimens. Immediately after the F-T tests were finished, all of the specimens in the testing set were provided with a central notch and subjected to the fracture tests. 


\subsubsection{Fracture test}

The fracture tests were performed in a three-point bending test configuration using a multipurpose stiff testing machine LaborTech 250 equipped with an electrical output enabling continuous recording of the loading force. The span length was set to $300 \mathrm{~mm}$ in all cases. The specimens were loaded with a constant displacement increment of $0.02 \mathrm{~mm} / \mathrm{min}$ during the whole time of the test duration. The deflection was continuously recorded in the middle of the span using an inductive sensor mounted in a special frame placed on the upper surface of the specimens. The crack mouth opening displacement was simultaneously recorded using a strain gauge mounted on the bottom part of the test specimens (see Fig. 2).

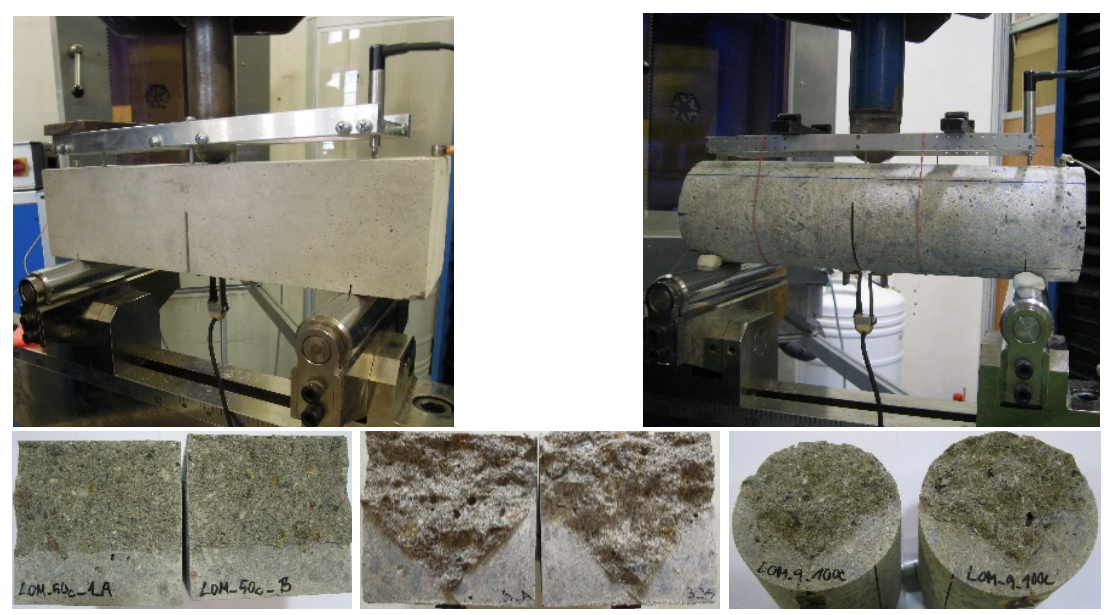

Fig. 2. Arrangement of the fracture tests and fracture surface after the test.

\section{Evaluation of the fracture tests}

\subsection{Fracture toughness}

The fracture toughness value was determined based on the linear elastic fracture mechanics approach for brittle fracture. This parameter is related to the stress field near the tip of the crack. The most commonly used specimen shape for determination of fracture toughness of concrete is a prismatic specimen with a central edge notch tested in the three-point bending configuration. The fracture toughness value $K_{\mathrm{Ic}}$ is calculated using this formula [10]:

$$
K_{\mathrm{I} c}=\frac{6 M_{\max }}{B W^{2}} Y(\alpha) \sqrt{a}
$$

where $M_{\max }$ is bending moment due to the maximum load and self-weight, $B$ is specimen's width, $W$ is specimen's depth, $Y(\alpha)$ is a function of geometry [7] and $a$ is notch depth.

In this case, a function of geometry proposed by Brown a Srawley (1966) was used [7]:

$$
Y(\alpha)=A_{0}+A_{1} \alpha+A_{2} \alpha^{2}+A_{3} \alpha^{3}+A_{4} \alpha^{4}
$$

Coefficients $A_{i}$ for the specimen in three-point bending were used according to [10], $\alpha=a / W$. 
Because the cylindrical specimens are not commonly used for the fracture tests of the concrete, the value of fracture toughness was determined in compliance with the recommendations given for testing of the fracture parameters of the rocks [9]:

$$
K_{\mathrm{I} c}=A_{\min } \frac{F_{\max }}{D^{1.5}}
$$

where $F_{\max }$ is the maximum load, $D$ is the specimen diameter, $A_{\min }$ is a geometry factor [9]:

$$
A_{\min }=\frac{S}{D} \cdot\left[1.835+7.15 \cdot \frac{a_{0}}{D}+9.85 \cdot\left(\frac{a_{0}}{D}\right)^{2}\right]
$$

where $S$ is the span length, $a_{0}$ is a Chevron V tip position.

\subsection{Modulus of elasticity}

The modulus of elasticity for the prismatic specimen with central edge notch tested in the three-point bending configuration was determined according to [11]:

$$
E_{c}=\frac{F_{i}}{4 B d_{i}}\left(\frac{S}{W}\right)^{3}\left[1-0.387 \frac{W}{S}+12.13\left(\frac{S}{W}\right)^{2.5}\right]+\frac{9}{2} \frac{F_{i}}{B d_{i}}\left(\frac{S}{W}\right)^{2} F_{1}(\alpha)
$$

where $F_{i}$ is load in the ascending linear part of the diagram; $d_{i}$ is the deflection at midspan corresponding with load $F_{i}, F_{1}(\alpha)=\int_{0}^{\alpha} x Y^{2}(x) d x$ and $Y(x)$ is the geometry function for the three-point bending configuration (2) [10].

For the cylindrical specimen with Chevron notch the modulus of elasticity was determined according to [9]:

$$
E_{c}=g_{0} \cdot \mathrm{s}_{\text {intial,tangent }} / \mathrm{D}
$$

where $s_{\text {initial,tangent }}$ is the initial tangent slope of the load vs deflection diagram, $g_{0}$ is a function of geometry [9]:

$$
g_{0}=20.8+19.4 \cdot \frac{a_{0}}{D}+142.3 \cdot\left(\frac{a_{0}}{D}\right)^{2}
$$

\subsection{Specific fracture energy}

The complete load vs deflection diagrams, including their post-peak parts, were employed to determine the work of fracture $W_{F, m}$, which is given by the area under the diagram. After that, the specific fracture energy values were determined according to the RILEM method [12]:

$$
G_{F, m}=\frac{W_{F, m}}{A_{\text {lig }}}
$$

where $A_{\text {lig }}$ is the area of the initially uncracked ligament, Fig. 1 introduces the ligament area for individual specimens. 


\subsection{Effective fracture toughness}

Several adaptations of linear elastic fracture mechanics have been proposed to take into account the nonlinear behaviour in an approximate manner. One representative is an effective crack model $[10,13]$, which includes the effect of pre-peak nonlinear behaviour of a real concrete structure containing initial notch through an equivalent elastic structure containing a notch of effective length $a_{e}>a$. The effective crack length is calculated from the secant stiffness of the concrete specimen at the peak load. The effective crack length $a_{e}$ for the prismatic specimen with a central edge notch tested in the three-point bending configuration was determined according to [10]:

$$
\begin{aligned}
d_{F_{\max }}=\frac{F_{\max }}{4 B E_{c}}\left(\frac{S}{W}\right)^{3}\left[1+\frac{5 q S}{8 F_{\max }}+\left(\frac{W}{S}\right)^{2}\left\{2.70+1.35 \frac{q S}{F_{\max }}\right\}-0.84\left(\frac{W}{S}\right)^{3}\right] \\
+\frac{9}{2} \frac{F_{\max }}{B E_{c}}\left(1+\frac{q S}{2 F_{\max }}\right)\left(\frac{S}{W}\right)^{2} F_{1}\left(\alpha_{e}\right)
\end{aligned}
$$

Subsequently, the effective fracture toughness value was calculated using a linear elastic fracture mechanics formula (1) where $\alpha$ was replaced with $\alpha_{e}=a_{e} / W$ in compliance with [10].

\section{Results and discussion}

The results of the fracture tests are shown in Figs. 3-5. Concerning the specific fracture energy, the absolute values determined on the reference sets of specimens is $105-145$ $\mathrm{J} / \mathrm{m}^{2}, 127-149 \mathrm{~J} / \mathrm{m}^{2}$ for the sets of specimens subjected to $50 \mathrm{~F}-\mathrm{T}$ cycles, and $117-147$ $\mathrm{J} / \mathrm{m}^{2}$ for the sets of specimens subjected to $100 \mathrm{~F}-\mathrm{T}$ cycles. Based on the results, it can be stated that the ratio of the absolute values of the fracture energy of the particular testing sets (non-frost attacked and frost-attacked) corresponds to the ratio of the area of the specimens' ligament in all cases. The trend of the development of the absolute values of fracture energy for prismatic specimens provided with the Chevron notch differs from the trend recorded for the other two sets of specimens (see Fig. 3).
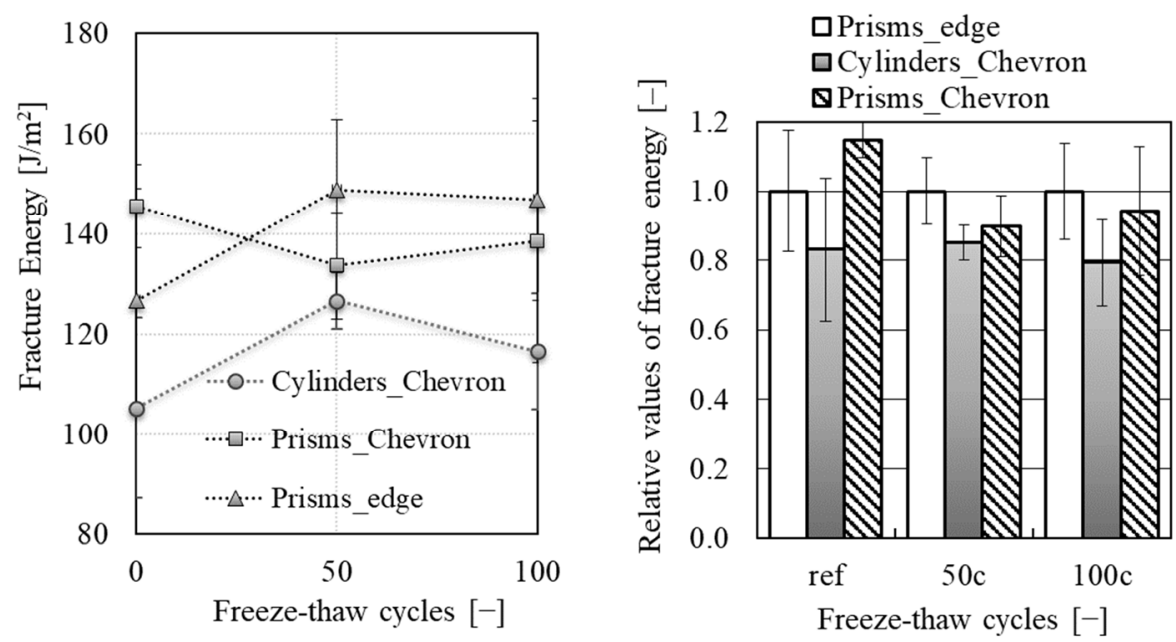

Fig. 3. Specific fracture energy. 
In the case of the modulus of elasticity and fracture toughness values, the correspondence of the ratio between the absolute values determined for the particular set of specimens and areas of the specimens' ligament is not observed. Because the geometry function for the prismatic specimens with a Chevron notch made from quasi-brittle materials has not been completed yet, the values of modulus of elasticity and fracture toughness were calculated only for the cylindrical specimens with the Chevron notch and prismatic specimens with the edge notch. Based on the results, it can be stated that the absolute values of the fracture toughness are sensitive to the shape of the specimens subjected to the F-T cycles. The ratio of the absolute values of fracture toughness determined for two different shapes of specimens differ in particular stages of the F-T test (see Table 1 and Fig. 5). Also, a strong influence of the numerical approach in the determination of the fracture toughness is observed in this particular case. If the non-linear behaviour is taken into account and an effective crack model is used for the calculation, the absolute value of the fracture toughness is $1.6-2.1$ times higher than the values determined based on the linear elastic fracture mechanics approach (see Fig. 5 - prisms with the edge notch).
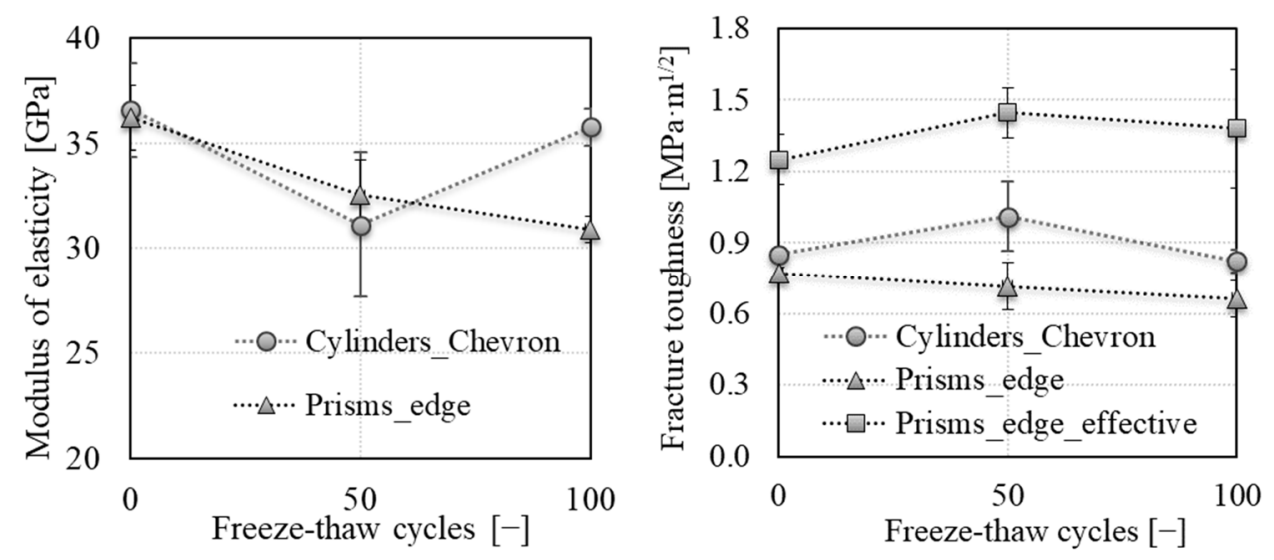

Fig. 4. Modulus of elasticity (left) and fracture toughness.
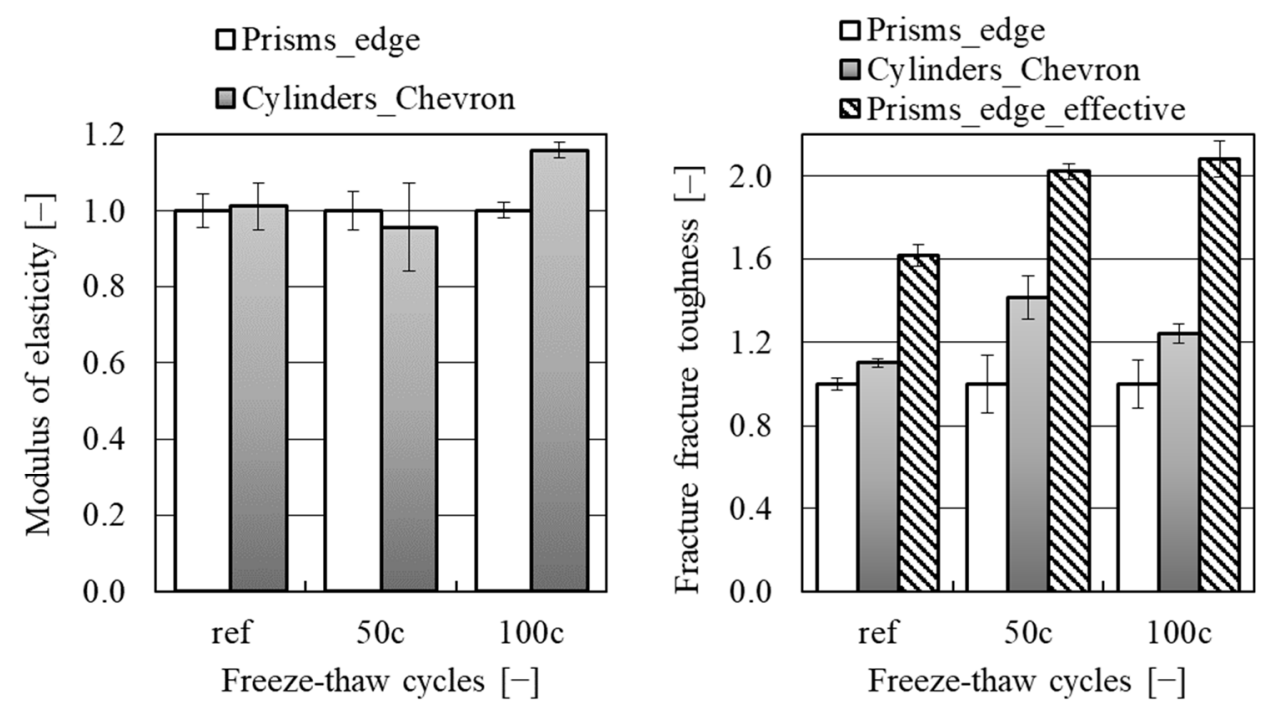

Fig. 5. Relative values of modulus of elasticity (left) and fracture toughness. 
Table 1. Relative values of mechanical fracture characteristics: Prismatic specimens with edge notch $=1$ (Relative coefficient of variability)

\begin{tabular}{|c|c|c|c|c|}
\hline Specimen set & $\boldsymbol{E}_{\boldsymbol{c}}[-]$ & $\boldsymbol{G}_{\boldsymbol{F}, \mathbf{m}}[-]$ & $\boldsymbol{K}_{\mathbf{I c}}[-]$ & \multirow{2}{*}{$\mathbf{A}[-]$} \\
\hline Cylindrical_Chevron_ref & $1.01(0.06)$ & $0.83(0.2)$ & $1.10(0.02)$ & \multirow{2}{*}{0.80} \\
\cline { 1 - 4 } Cylindrical_Chevron_50c & $0.96(0.11)$ & $0.85(0.05)$ & $1.41(0.10)$ & \\
\cline { 1 - 4 } Cylindrical_Chevron_100c & $1.16(0.02)$ & $0.79(0.12)$ & $1.24(0.05)$ & \\
\hline
\end{tabular}

\section{Conclusion}

The paper presents the results of the pilot study focused on the determination of the fracture characteristics of the concrete exposed to the F-T cycles. The influence of the shape of specimens and the initial notch on the absolute value of fracture characteristics were investigated experimentally. Three types of specimens were used in the experimental program - prisms with edge and Chevron notch, and cylinders with the Chevron notch. Based on the results, it can be stated that the values of fracture energy are proportional to the area of the ligament. This finding was observed for all tested sets - non-frost and frost attacked. On the other hand, it seems that the values of modulus of elasticity and fracture toughness are more influenced by the geometry than only by the area of ligament and also by the changes of the specimens' stiffness due to the exposition to the F-T cycles. More tests need to be performed to confirm this hypothesis. Also, a strong influence of the numerical approach in the determination of the fracture toughness was observed. The wide difference between the results obtained from the linear elastic fracture mechanics approach for brittle and quasi-brittle materials was observed. Currently, the fracture toughness and modulus of elasticity for the prismatic specimens with the Chevron notch cannot be calculated because of missing a suitable geometry function.

This work has been supported by the Czech Science Foundation, project No. 19-22708S.

\section{References}

1. ASTM C666 / C666M-15 (2015)

2. CEN/TR 15177: (2006)

3. G. Wardeh, E. Ghorbel, Proc. of IA-FraMCoS, (2013)

4. P. Qiao, F. Chen, Constr. Build. Mater. 47, (2013)

5. Y. Dong, Ch. Su, P. Qiao, L. Z Sun, Int. J. Damage Mech. 27, 8 (2018)

6. P. Miarka, L. Pan, V. Bílek, H. Cifuentés, S. Seitl, Proc. of IA-FraMCoS (2019)

7. A. Momeni, Y. Abdilor, G. R. Khanlari, M. Heidari, A. A. Sepahi, B. Eng. Geol. Environ. 75, 4 (2016)

8. ČSN 731322: (1968)

9. F. Ouchterlony, Int. J. Rock. Mech. Min. 25, 2 (1988)

10. B. L. Karihaloo, Fracture mechanics and structural concrete (Longman Scientific \& Technical, Harlow, Essex, England, 1995)

11. M. Stibor, Fracture Parameters of Quasi-Brittle Materials and Their Determination, dissertation thesis, (2004) (in Czech) 
12. RILEM TC - 50 FMC (Recommendation). Mater. Struct. 18, 4 (1985)

13. P. Nallathambi, B.L. Karihaloo, Mag. Concrete Res. 38, 135 (1986) 